Numer. Funct. Anal. Optim , 23(5\&6), 477-497 (2002)

\title{
Nearness, Accretivity and the Solvability of Nonlinear Equations
}

\author{
Adriana Buica ${ }^{1}$ and Andras Domokos ${ }^{1,2}$ \\ ${ }^{1}$ Babes-Bolyai University, Department of Applied \\ Mathematics, str. M. Kogalniceanu 1, 3400 \\ Cluj-Napoca, Romania \\ ${ }^{2}$ Department of Mathematics, University of \\ Pittsburgh, Pittsburgh, PA 15260, USA
}

\begin{abstract}
In this paper our propose is to find a common term which is included in the assumptions of theorems proving existence of zeros, implicit functions, fixed points or coincidence points. This new point of view allows us to weaken the assumptions which guarantee the solvability of nonlinear equations and to recommend a possible unified treatment of several classes of operators which appear in the theory of nonlinear equations.
\end{abstract}

\section{Introduction}

In general, to prove the existence of zeros, of implicit functions, fixed points or coincidence points, one can use either assumptions in con-

www.dekker.com 
nection with the geometrical properties of Banach spaces or the nearness of a mapping having good properties. By assumptions which are in connection with the geometrical properties of the Banach spaces, we mean monotonicity $[2,18,36]$, accretivity $[5,6,17,19,27,28,30]$, $K$-positivity $[14,16]$, pseudo-contractivity $[12,22,26])$. The nearness of two mappings $A$ and $B$ is satisfied, for example, when $B$ is the differential of $A$ at a point and $A$ is a $C^{1}$-mapping or in the nonsmooth case if $B$ strongly approximates $A$ in the sense of Robinson $[20,31,32]$ or $B$ is near $A$ in the sense of Campanato [10, 11, 34, 35]. We will show that these assumptions have the same origin, which is a generalized accretivity. In this way the iterative procedures for finding zeros of strongly accretive mappings [13, 15, 24, 25] and fixed points for strong pseudo-contractions $[13,38]$ can be used to approximate the solutions of a great variety of nonlinear equations.

We start with a well-known nearness property of the differential. Let $X$ and $Z$ be Banach spaces, $x_{0} \in X, X_{0}$ its neighborhood. Let $A: X_{0} \rightarrow Z$ be a mapping which is Fréchet-differentiable on $X_{0}$ and suppose that $d A: X_{0} \rightarrow \mathcal{L}(X, Z)$ is continuous at $x_{0}$. Then $d A\left(x_{0}\right)$ approximates $A$ in a neighborhood of $x_{0}$ in the following sense: for all $\varepsilon>0$ there exists a neighborhood $X_{\varepsilon} \subset X_{0}$ of $x_{0}$ such that

$$
\left\|d A\left(x_{0}\right)(x-y)-(A x-A y)\right\| \leq \varepsilon\|x-y\|, \forall x, y \in X_{\varepsilon} .
$$

This property of the $d A\left(x_{0}\right)$ was generalized by Robinson [31] for nonsmooth mappings. Using Robinson's idea, nonsmooth implicit function and inverse function theorems were proved [19, 20, 32].

We recall now the definition of the strong approximation given by Robinson [31].

Definition I.1 Let $X$ and $Z$ be Banach spaces, $x_{0} \in X$ and $X_{0}$ its neighborhood. Let $A, B: X_{0} \rightarrow Z$. We say that $A$ strongly approximates $B$ at $x_{0}$ if for all $\varepsilon>0$ there exists a neighborhood $X_{\varepsilon} \subset X_{0}$ of $x_{0}$ such that for all $x, y \in X_{\varepsilon}$

$$
\|B(x)-B(y)-(A(x)-A(y))\| \leq \varepsilon\|x-y\| .
$$

The following notion of nearness was given by Campanato [10, 34].

Definition I.2 Let $A$ and $B$ be two mappings from a set $X$ into a 
Banach space $Z$. We say that $A$ is near $B$ if there exist $\alpha>0$ and $0 \leq c<1$ such that for all $x, y \in X$ we have

$$
\|B x-B y-\alpha(A x-A y)\| \leq c\|B x-B y\| .
$$

Remark I.1 A. Tarsia proved ([34], Theorem 3.2$)$ that in the context of Definition 1.1, if for some $\varepsilon>0,\left.B\right|_{X_{\varepsilon}}: X_{\varepsilon} \rightarrow B\left(X_{\varepsilon}\right)$ is invertible such that its inverse is Lipschitz continuous and if $A$ strongly approximates $B$ at $x_{0}$, then $A$ is near $B$ in a neighborhood of $x_{0}$. We notice that for this implication it would be enough to suppose that (1.1) holds for some $\varepsilon>0$ with $\varepsilon \cdot l<1$, where $l$ is the Lipschitz constant of the inverse of $\left.B\right|_{X_{\varepsilon}}$.

In order to prove connections between nearness and accretivity let us introduce the following definitions. These definitions are generalizations of the well-known notions of accretivity, strong or $\varphi$-accretivity. First we define a class $\Phi$ of functions $\varphi: \mathbb{R}_{+} \rightarrow \mathbb{R}_{+}$which will be used to define the $\varphi$-accretivity. We say that $\varphi \in \Phi$ if $\varphi(0)=0$, $\varphi(r)>0$ for $r>0, \liminf _{r \rightarrow \infty} \varphi(r)>0$ and $\liminf _{r \rightarrow r_{0}} \varphi(r)=0$ implies $r_{0}=0$.

In this class belong continuous mappings $\varphi: \mathbb{R}_{+} \rightarrow \mathbb{R}_{+}$with $\varphi(0)=$ $0, \varphi(r)>0$ for $r>0$ and $\liminf \operatorname{in}_{r \rightarrow \infty} \varphi(r)>0([17,25,28])$ and nondecreasing mappings $\varphi$ with $\varphi(r)>0$ for $r>0([2,19,37])$.

We remind that in a Banach space $Z$ the normalized duality mapping $J: Z \leadsto Z^{*}$ is defined by

$$
J(z)=\left\{z^{*} \in Z^{*}:\left\langle z^{*}, z\right\rangle=\left\|z^{*}\right\| \cdot\|z\|=\|z\|^{2}\right\} .
$$

Definition I.3 Let $A$ and $B$ be two mappings from a set $X$ into a Banach space $Z$ and $J: Z \leadsto Z^{*}$ be the normalized duality mapping of $Z$.

a) We say that $A$ is accretive with respect to $B$ if for all $x, y \in X$ there exists $j(B x-B y) \in J(B x-B y)$ such that

$$
\langle A x-A y, j(B x-B y)\rangle \geq 0 .
$$

If instead of (1.3) we suppose

$$
\langle A x-A y, j(B x-B y)\rangle \geq c\|B x-B y\|^{2}
$$

for some $0<c<1$, then we say that $A$ is strongly accretive with 
respect to $B$.

If instead of (1.3) we suppose

$$
\langle A x-A y, j(B x-B y)\rangle \geq \varphi(\|B x-B y\|)\|B x-B y\|
$$

where $\varphi \in \Phi$, then we say that $A$ is $\varphi$-accretive with respect to $B$. b) We say that $A$ is Lipschitzian with respect to $B$ if there exists $L>0$ such that for all $x, y \in X$

$$
\|A x-A y\| \leq L\|B x-B y\| .
$$

Remark I.2 When $Z$ is a Hilbert space, then the above accretivity notions coincide with the monotonicity, strong monotonicity ([11, $35])$ and $\varphi$-monotonicity with respect to $B$. Moreover, if in addition we suppose that $B=I$ we get the notions of monotonicity, strong monotonicity and $\varphi$-monotonicity $([2,3,4,37])$.

When $X=Z$ is a Banach space and $B=I$ we get the well-known accretivity, strong accretivity and $\varphi$-accretivity $([5,13,15,17,18$, $19,24,25,27,28,30,37,38])$.

In the case of linear mappings $A=L, B=K: Z \rightarrow Z$ we get the definition of the $K$-positive definite operator $([14,16])$, namely

$$
\langle A x, j(B x)\rangle \geq c\|B x\|^{2} .
$$

In Section 2 we define the notion of weak-nearness between two operators using the accretivity condition (1.5). We will prove that some properties (injectivity, surjectivity, openess, homeomorphism) carry over to weakly-near operators.

The main results of Section 3 prove the existence of zeros and of implicit functions for $A x=0$ and $A(x, \lambda)=0$ respectively, when $A$ is weakly-near to some "good" mapping $B$.

In Section 4 we consider the solvability of a Dirichlet problem for certain types of fully nonlinear elliptic equations, including those which cannot be linearized. Our treatment differs from the methods used for finding viscosity solutions for fully nonlinear elliptic equations, by the fact that we use the geometrical properties of Banach spaces to measure the nearness between functions, instead of focusing on the ordering relations which are needed to have viscosity subsolutions and supersolutions (see [9]). 


\section{The notion of weak-nearness}

A. Tarsia [34] and S. Campanato [11] mentioned that in the case of a Hilbert space $Z, A$ is near $B$ if and only if $A$ is strongly monotone with respect to $B$ and Lipschitzian with respect to $B$. In Banach spaces we can only prove that nearness implies strong-accretivity and Lipschitz continuity.

Theorem II.1 Let $A$ and $B$ be two mappings from a set $X$ into a Banach space $Z$. If $A$ is near $B$, then $A$ is strongly accretive with respect to $B$ and Lipschitzian with respect to $B$.

Proof. Let us suppose that $A$ is near $B$, which means that there exist $\alpha>0,0<c<1$ such that for all $x, y \in X$

$$
\|B x-B y-\alpha(A x-A y)\| \leq c\|B x-B y\| .
$$

Hence, for each $j(B x-B y) \in J(B x-B y)$ we have

$$
\begin{gathered}
\langle B x-B y-\alpha(A x-A y), j(B x-B y)\rangle \leq \\
\leq\|B x-B y\|\|B x-B y-\alpha(A x-A y)\| \leq c\|B x-B y\|^{2},
\end{gathered}
$$

so

$\langle B x-B y, j(B x-B y)\rangle-\alpha\langle A x-A y, j(B x-B y)\rangle \leq c\|B x-B y\|^{2}$,

and

$$
\langle A x-A y, j(B x-B y)\rangle \geq \frac{1-c}{\alpha}\|B x-B y\|^{2} .
$$

If $\frac{1-c}{\alpha}<1$ then the proof is complete, otherwise we can choose instead of $\frac{1-c}{\alpha}$ an arbitrary positive constant less then 1 .

In this way we have proved that $A$ is strongly accretive with respect to $B$.

In order to prove that $A$ is Lipschitzian with respect to $B$ it is enough to notice that

$$
\begin{gathered}
\alpha\|A x-A y\|-\|B x-B y\| \leq \\
\leq\|B x-B y-\alpha(A x-A y)\| \leq c\|B x-B y\| .
\end{gathered}
$$


Remark II.1 For the completeness of our treatment, let us prove the reverse implication in the case of a Hilbert space $Z$.

Assuming that $A$ is strongly monotone and Lipschitzian with respect to $B$, for an $\alpha>0$ we obtain

$$
\begin{gathered}
\|B x-B y-\alpha(A x-A y)\|^{2}= \\
=\|B x-B y\|^{2}-2 \alpha\langle B x-B y, A x-A y\rangle+\alpha^{2}\|A x-A y\|^{2} \leq \\
\leq\left(1-2 \alpha c+L^{2} \alpha^{2}\right)\|B x-B y\|^{2} .
\end{gathered}
$$

We finish the proof by observing that there exists $\alpha>0$ such that $0<1-2 \alpha c+L^{2} \alpha^{2}<1$.

Remark II.2 From the proof of Theorem 2.1 we realize that the following two conditions are equivalent:

(i) There exists $\alpha>0$ and $0<c<1$ such that

$\langle B x-B y-\alpha(A x-A y), j(B x-B y)\rangle \leq c\|B x-B y\|^{2}, \forall x, y \in X$.

(ii) $A$ is strongly accretive with respect to $B$.

In the following definition we give a notion which is weaker than the Lipschitz continuity with respect to $B$.

Definition II.1 Let $A$ and $B$ be two mappings from a set $X$ to a Banach space $Z$. We say that $A$ is continuous with respect to $B$ if $A \circ B^{-1}: B(X) \leadsto Z$ has a continuous selection.

If $A$ is Lipschitzian with respect to $B$, then $A$ is continuous with respect to $B$. Indeed, in this case $A \circ B^{-1}: B(X) \rightarrow Z$ is single-valued and Lipschitz continuous, even if $B^{-1}$ is not single valued.

We will introduce a weaker notion of nearness than those from Definitions 1.1 and 1.2 and we will use it to prove the existence of zeros, the existence of implicit functions and the existence of solutions for fully nonlinear elliptic equations.

Definition II.2 Let $A$ and $B$ be two mappings from a set $X$ into a Banach space $Z$. We say that $A$ is weakly-near $B$ if $A$ is $\varphi$-accretive with respect to $B$ and continuous with respect to $B$. 
Let us notice that, if $X=Z$ and $A: Z \rightarrow Z$ is $\varphi$-accretive and continuous, then $A$ is weakly-near to $I$, the identity map of $Z$.

Using Theorem 2.1, we can see that if $A$ is near $B$ then $A$ is weakly near $B$. In order to have the weak-nearness a strictly weaker condition than the nearness we need either a non Hilbert Banach space $Z$, or a weaker accretivity than the strong accretivity, or continuity instead Lipschitz continuity.

A. Tarsia mentioned in [35] that if $A$ is near $B$ then $A x=A y$ if and only if $B x=B y$. When $A$ is weakly-near $B$ we have only that $A x=A y$ implies $B x=B y$.

In [35] it is proved that some properties as injectivity, surjectivity, openess are preserved by nearness. In the next proposition we state that they are also preserved by weak-nearness.

Proposition II.1 Let $A, B: X \rightarrow Z$ be such that $A$ is weakly-near $B$. If $B$ is injective then $A$ is injective and, if $B$ is surjective, then $A$ is surjective.

If, in addition, we suppose that $B(X)$ is open and

(i) $B x=B y$ implies that $A x=A y$, then $A(X)$ is open. In the case of a topological space $X$, if $B$ is a homeomorphism, then $A$ is a homeomorphism, too.

Proof. Let $B$ be injective. In order to prove that $A$ is injective, let us consider $x, y \in X$ with $A x=A y$. Using (1.5) we get that $B x=B y$, which implies that $x=y$.

Let $g: B(X) \rightarrow Z$ be the continuous selection of $A \circ B^{-1}$. Since $A$ is weakly-near $B$ it follows that $g$ is $\varphi$-accretive. To prove this, let $u, v \in B(X)$. Then $g(u) \in A \circ B^{-1}(u)$ and $g(v) \in A \circ B^{-1}(v)$ which means that there exist $x \in B^{-1}(u)$ and $y \in B^{-1}(v)$ such that $g(u)=$ $A x, u=B x, g(v)=A y$ and $v=B y$. Then

$$
\langle A x-A y, j(B x-B y)\rangle \geq \varphi(\|B x-B y\|)\|B x-B y\|
$$

implies that

$$
\langle g(u)-g(v), j(u-v)\rangle \geq \varphi(\|u-v\|)\|u-v\| .
$$

Now let us suppose that $B$ is surjective. Since the mapping $g: Z \rightarrow Z$ is $\varphi$-accretive and continuous, it is also surjective ([28], Theorem 3 ). 
Hence $A \circ B^{-1}$ is surjective, which implies that $A$ is surjective. Assumption $(i)$ implies that $A \circ B^{-1}$ is single-valued, therefore $g=$ $A \circ B^{-1}$. Moreover, $g(B(X))=A(X)$. Hence if $B(X)$ is open, then $g(B(X))$ is open, too ([28], Corollary 3).

Let us consider now that $B$ is a homeomorphism. Using what we have just proved regarding the injectivity and surjectivity of $A$, we deduce that $A$ is bijective.

Since $A$ is weakly-near $B$, it follows that $A \circ B^{-1}$ is continuous. Using the continuity of $B$ and that $A=A \circ B^{-1} \circ B$, we get that $A$ is continuous.

From (1.5) we obtain that

$$
\|A x-A y\| \geq \varphi(\|B x-B y\|) .
$$

Hence

$$
\varphi\left(\left\|B \circ A^{-1} u-B \circ A^{-1} v\right\|\right) \leq\|u-v\|
$$

which implies that $B \circ A^{-1}$ is continuous. Using $A^{-1}=B^{-1} \circ B \circ$ $A^{-1}$ and the continuity of $B^{-1}$, we deduce that $A^{-1}$ is continuous.

\section{Existence of zeros and of implicit functions by using the weak-nearness}

First we will recall a lemma regarding the existence of zeros in Banach spaces for $\varphi$-accretive mappings. We will use this lemma to prove the existence of zeros for mappings which act between a set and a Banach space. Actually, these two results are equivalent, because if in Theorem 3.1 we take $X=\mathcal{B}\left(z_{0}, r\right)$ and $B=I$ we get Lemma 3.1.

By $\mathcal{B}\left(z_{0}, r\right)$ we denote the closed ball centered at $z_{0}$ and of radius $r>0$.

Lemma III.1 $[19,27]$ Let $Z$ be a Banach space, $z_{0} \in Z$ and $r>0$. Let us suppose that there is a continuous, $\varphi$-accretive mapping $g$ : 
$\mathcal{B}\left(z_{0}, r\right) \rightarrow Z$ such that for all $z \in \partial \mathcal{B}\left(z_{0}, r\right)$ and $t>0$ holds

$$
0 \neq t\left(z-z_{0}\right)+g(z) .
$$

Then there exists $\bar{z} \in \mathcal{B}\left(z_{0}, r\right)$ such that $g(\bar{z})=0$.

Theorem III.1 Let $X$ be a set, $Z$ be a Banach space, $z_{0} \in Z$ and $A, B: X \rightarrow Z$. Let us suppose that:

(i) $\mathcal{B}\left(z_{0}, r\right) \subset B(X)$,

(ii) $A$ is weakly-near to $B$,

(iii) $0 \neq t\left(B x-z_{0}\right)+A x, \forall t>0$ and $x \in X$ with $\left\|B x-z_{0}\right\|=r$. Then there exists $\bar{x} \in X$ such that $A \bar{x}=0$ and $\left\|B \bar{x}-z_{0}\right\| \leq r$.

Proof. Let $g: B(X) \rightarrow Z$ be the continuous selection of $A \circ B^{-1}$. Since $A$ is weakly-near to $B$, it follows that $g$ is $\varphi$-accretive (in the same manner as in the proof of Proposition 2.1). Assumption (iii) implies that

$$
0 \neq t\left(z-z_{0}\right)+g(z)
$$

for all $t>0$ and $z \in \partial \mathcal{B}\left(z_{0}, r\right)$.

So the mapping $\left.g\right|_{\mathcal{B}\left(z_{0}, r\right)}$ satisfies the hypothesis of Lemma 3.1, and hence there exists $\bar{z} \in \mathcal{B}\left(z_{0}, r\right)$ with $g(\bar{z})=0$.

Therefore, $0 \in A \circ B^{-1}(\bar{z})$, which implies the existence of $\bar{x} \in B^{-1}(\bar{z})$ such that $A(\bar{x})=0$.

We will prove now an implicit function theorem, which can be considered a generalization of the classical implicit function theorem and of the implicit function theorems found in [19, 20, 31, 32, 34]. The main advantage of this theorem is that we do not assume a uniform $\varphi$-accretivity of the mappings $A(\cdot, \lambda)$ with respect to $B$ and that the set $X$ does not need to have any linear or topological properties. (see Corollary 3.1 and the connections with other implicit function theorems [19]).

Theorem III.2 Let $X$ be a set, $\Lambda$ be a topological space, $Z$ be a Banach space. We consider $x_{0} \in X, \lambda_{0} \in \Lambda, z_{0} \in Z$, a neighborhood $\Lambda_{0}$ of $\lambda_{0}$, a constant $r>0$ and the mappings $A: X \times \Lambda_{0} \rightarrow Z, B$ : $X \rightarrow Z$ such that

(i) $x_{0} \in B^{-1}\left(z_{0}\right)$. 
(ii) $\mathcal{B}\left(z_{0}, r\right) \subset B(X)$,

(iii) $A(\cdot, \lambda)$ is weakly-near to $B$ (with respect to a function $\varphi_{\lambda}$ ) for all $\lambda \in \Lambda_{0} \backslash\left\{\lambda_{0}\right\}$.

(iv) $\lim _{\lambda \rightarrow \lambda_{0}} \inf \left\{R>0: \varphi_{\lambda}(R)-\left\|A\left(x_{0}, \lambda\right)\right\|>0\right\}=0$.

Then there exist a neighborhood $\Lambda_{1}$ of $\lambda_{0}$ and a mapping $x: \Lambda_{1} \backslash$ $\left\{\lambda_{0}\right\} \rightarrow X$ such that for all $\lambda \in \Lambda_{1} \backslash\left\{\lambda_{0}\right\}$ we have $A(x(\lambda), \lambda)=0$ and

$$
\lim _{\lambda \rightarrow \lambda_{0}}\left\|B(x(\lambda))-B\left(x_{0}\right)\right\|=0 .
$$

Proof. Let

$$
\Lambda_{1}=\left\{\lambda \in \Lambda_{0}: \inf \left\{R>0: \varphi_{\lambda}(R)-\left\|A\left(x_{0}, \lambda\right)\right\|>0\right\}<r\right\}
$$

and $\lambda \in \Lambda_{1} \backslash\left\{\lambda_{0}\right\}$. Since

$\varphi_{\lambda}\left(\left\|B x-B x_{0}\right\|\right)\left\|B x-B x_{0}\right\| \leq\left\langle A(x, \lambda)-A\left(x_{0}, \lambda\right), j\left(B x-B x_{0}\right)\right\rangle$,

and for all $t \geq 0$ we have

$$
0 \leq\left\langle t\left(B x-B x_{0}\right), j\left(B x-B x_{0}\right)\right\rangle,
$$

it follows that

$$
\begin{gathered}
\varphi_{\lambda}\left(\left\|B x-B x_{0}\right\|\right)\left\|B x-B x_{0}\right\| \leq \\
\leq\left\langle A(x, \lambda)+t\left(B x-B x_{0}\right), j\left(B x-B x_{0}\right)\right\rangle-\left\langle A\left(x_{0}, \lambda\right), j\left(B x-B x_{0}\right)\right\rangle . \\
\text { If }\left\|B x-B x_{0}\right\|=\left\|B x-z_{0}\right\|=R, \text { then } \\
\left.\qquad A(x, \lambda)+t\left(B x-B x_{0}\right), j\left(B x-B x_{0}\right)\right\rangle \geq \\
\geq \varphi_{\lambda}(R) R+\left\langle A\left(x_{0}, \lambda\right), j\left(B x-B x_{0}\right)\right\rangle
\end{gathered}
$$

and hence

$$
\left\|A(x, \lambda)+t\left(B x-z_{0}\right)\right\| \geq \varphi_{\lambda}(R)-\left\|A\left(x_{0}, \lambda\right)\right\| .
$$

Assumption (iv) implies that for each $\lambda \in \Lambda_{1} \backslash\left\{\lambda_{0}\right\}$ we can choose $R_{\lambda} \in[0, r]$ such that $\varphi_{\lambda}\left(R_{\lambda}\right)-\left\|A\left(x_{0}, \lambda\right)\right\|>0$ and $R_{\lambda} \rightarrow 0$ as $\lambda \rightarrow$ $\lambda_{0}$. Using Theorem 3.1 we conclude that for all $\lambda \in \Lambda_{1} \backslash\left\{\lambda_{0}\right\}$ there exists $x(\lambda) \in X$ such that $A(x(\lambda), \lambda)=0$ and $\left\|B(x(\lambda))-B\left(x_{0}\right)\right\| \leq$ $R_{\lambda}$. 
The following corollary is a special case of Theorem 3.2 in the following setting: $X=Z, A=g, B=I, \varphi_{\lambda}=\varphi$.

Corollary III.1 [19] Let $Z$ be a Banach space, $\Lambda$ be a normed space, $\left(x_{0}, \lambda_{0}\right) \in Z \times \Lambda$. Let us suppose that there exist neighborhoods $Z_{0}$ of $z_{0}, \Lambda_{0}$ of $\lambda_{0}$ and a mapping $g: Z_{0} \times \Lambda_{0} \rightarrow Z$ such that

(i) $0=g\left(z_{0}, \lambda_{0}\right)$;

(ii) the mappings $g(., \lambda)$ are continuous on $Z_{0}$, for all $\lambda \in \Lambda_{0}$;

(iii) the mappings $g(., \lambda)$ are $\varphi$-accretive on $Z_{0}$ (with respect to the same function $\varphi)$, for all $\lambda \in \Lambda_{0}$;

(iv) $g\left(z_{0},.\right)$ is continuous at $\lambda_{0}$.

Then there exists a neighborhood $\Lambda_{1}$ of $\lambda_{0}$ and a unique mapping $x$ : $\Lambda_{1} \rightarrow Z_{0}$ such that $z\left(\lambda_{0}\right)=z_{0}, z$ is continuous at $\lambda_{0}$ and $g(z(\lambda), \lambda)=$ 0 for all $\lambda \in \Lambda_{1}$.

\section{Fully nonlinear elliptic PDE}

In this section we consider the solvability of the Dirichlet problem for certain types of fully nonlinear elliptic equations. For these problems we use the method of weakly-near operators. We obtain existence and uniqueness results and, also, $W^{2, p}$-estimates for the solutions.

The theory of near operators was applied by S. Campanato [11] and A. Tarsia $[34,35]$ to fully nonlinear elliptic systems. They proved that the ellipticity condition (4.1) implies that the operator $A$ (see Theorem 4.1 below) is near the Laplace operator $\Delta$ in $L^{2}(\Omega)$.

We will use the ellipticity condition (4.7) to prove that a more difficult operator $A$, given by (4.11), is weakly-near to the linear operator $B$, given by (4.5), in $L^{p}(\Omega)$.

The ellipticity condition (4.1) implies that the function $a$ is Lipschitz with respect to $M$. This fact assures that $M \rightarrow a(x, M)$ is almost everywhere differentiable, which allows the linearization of equation (4.2). Our results permit to deal also with equations for which the linearization is not possible, since our function $a(x, \cdot)$ need not to be differentiable or Lipschitz.

In what follows, $\Omega$ will be a $C^{2}$ bounded domain of $\mathbb{R}^{n}$. 
We denote by $\mathbb{R}^{n \times n}$ the space of $n \times n$ real matrices; $|\cdot|_{m}$ is the Euclidian norm in $\mathbb{R}^{m} ; \operatorname{tr} N=\sum_{i=1}^{n} \xi_{i i}$ is the trace of the $n \times n$ matrix $N=\left(\xi_{i j}\right)$.

In $[11,34,35]$ the authors considered a function $a: \Omega \times \mathbb{R}^{n \times n} \rightarrow \mathbb{R}$ such that

(i) $a(x, 0)=0$,

(ii) $a(\cdot, M)$ is measurable,

(iii) $a(x, \cdot)$ is continuous,

(iv) there exists three positive constants $\alpha, \beta, \gamma$, with $\gamma+\delta<1$ such that

$$
|\operatorname{tr} N-\alpha[a(x, M+N)-a(x, M)]| \leq \gamma|N|_{n^{2}}+\delta|\operatorname{tr} N|,
$$

for almost every $x \in \Omega$, for all $M, N \in \mathbb{R}^{n \times n}$.

The main result in [11] is the following.

Theorem IV.1 If all the above conditions hold then

$A(u)=a\left(x, D^{2} u\right)$ is a well-defined operator between $H^{2}(\Omega) \cap H_{0}^{1}(\Omega)$ and $L^{2}(\Omega)$. Moreover,

(i) $A$ is near $\Delta$

and, consequently,

(ii) $A$ is bijective.

This theorem implies that for every $f \in L^{2}(\Omega)$ the problem

$$
u \in H^{2}(\Omega) \cap H_{0}^{1}(\Omega), \quad a\left(x, D^{2} u\right)=f(x), \text { a.e. } x \in \Omega
$$

has a unique solution.

We remark that $a$ satisfies condition (4.1) if and only if

$$
\begin{aligned}
& {[a(x, M+N)-a(x, M)] \operatorname{tr} N \geq} \\
& \geq \frac{1-\delta}{\alpha}|\operatorname{tr} N|^{2}-\frac{\gamma}{\alpha}|\operatorname{tr} N||N|_{n^{2}} \\
& |a(x, M+N)-a(x, M)| \leq \frac{1+\delta}{\alpha}|\operatorname{tr} N|+\frac{\gamma}{\alpha}|N|_{n^{2}} .
\end{aligned}
$$

One could prove that $A$ is weakly-near $\Delta$ (see [8]) if we replace (4.1) by the weaker condition that there exist some positive constants $c_{i}$, 
$i \in\{1, \ldots, 5\}$ with $c_{1}>c_{2}+c_{3}$ such that

$$
\begin{aligned}
& {[a(x, M+N)-a(x, M)] \operatorname{tr} N \geq} \\
& \geq c_{1}|\operatorname{tr} N|^{2}-c_{2}|\operatorname{tr} N||N|_{n^{2}}-c_{3}|N|_{n^{2}}^{2}, \\
& |a(x, N)| \leq c_{4}|\operatorname{tr} N|+c_{5}|N|_{n^{2}} .
\end{aligned}
$$

Theorem 4.1 remains valid under (4.4).

In what follows we shall propose a condition, which assures that $A$ is weakly-near to some linear elliptic operator

$$
B u=\sum_{i, j=1}^{n} l_{i j}(x) \frac{\partial^{2} u}{\partial x_{i} \partial x_{j}}+\sum_{i=1}^{n} l_{i}(x) \frac{\partial u}{\partial x_{i}}-l_{0}(x) u
$$

where $L=\left(l_{i j}\right) \in C\left(\bar{\Omega}, \mathbb{R}^{n \times n}\right), l=\left(l_{i}\right) \in L^{\infty}\left(\Omega, \mathbb{R}^{n}\right), l_{0} \in L^{\infty}(\Omega)$, $l_{0} \geq 0$ and

$$
\sum_{i, j=1}^{n} l_{i j}(x) \xi_{i} \xi_{j} \geq \mu|\xi|^{2} \quad, \forall x \in \Omega, \xi \in \mathbb{R}^{n} .
$$

Moreover, we shall work in $L^{p}(\Omega)$, with $2 \leq p<\infty$.

Before stating our assumptions and results, let us write the operator $B$ and its ellipticity condition as (see [33]):

$$
\begin{gathered}
B u=\operatorname{tr}\left(L(x) D^{2} u\right)+l D u-l_{0} u, \\
\operatorname{tr}(L(x) N) \geq \mu \operatorname{tr} N, \quad \forall N \in S^{n}, N \geq 0,
\end{gathered}
$$

where $S^{n}$ denotes the set of $n \times n$ symmetrical matrices and $N \geq 0$ means that the symmetrical matrix $N=\left(\xi_{i j}\right)$ is semipositive definite (i.e. $\sum_{i, j=1}^{n} \xi_{i j} \eta_{i} \eta_{j} \geq 0$ for all $\left.\eta=\left(\eta_{1}, \cdots, \eta_{n}\right) \in \mathbb{R}^{n}\right)$.

We suppose that the function $a: \Omega \times \mathbb{R} \times \mathbb{R}^{n} \times \mathbb{R}^{n \times n} \rightarrow \mathbb{R}$ satisfies the following conditions:

(a1) $a(x, 0,0,0)=0$,

(a2) $a(\cdot, r, d, M)$ is measurable,

(a3) $a(x, \cdot, \cdot, \cdot)$ is continuous,

(a4) there exist $\alpha, \beta, \gamma \geq 0$ such that

$$
|a(x, r, d, M)| \leq \alpha|r|+\beta|d|_{n}+\gamma|M|_{n^{2}},
$$


$n<2 p$ and there exist $\alpha:[0, \infty) \rightarrow[0, \infty)$ continuous, $\beta, \gamma \geq 0$, $s \geq 1$ such that

$$
|a(x, r, d, M)| \leq \alpha(|r|)\left(\beta|d|_{n}^{s}+\gamma|M|_{n^{2}}\right),
$$

(a5) there exists $c>0$ such that

$$
\begin{gathered}
{[a(x, r+s, d+\delta, N+M)-a(x, s, \delta, M)] \cdot} \\
\cdot\left[\operatorname{tr}(L(x) N)+l(x) d-l_{0}(x) r\right] \geq \\
\geq c\left|\operatorname{tr}(L(x) N)+l(x) d-l_{0}(x) r\right|^{2},
\end{gathered}
$$

for almost all $x \in \Omega$, for all $r, s \in \mathbb{R}, d, \delta \in \mathbb{R}^{n}, M, N \in \mathbb{R}^{n \times n}$.

We can see that the ellipticity condition (a5) implies that the following ellipticity condition used in [33] is satisfied:

$$
a(x, s, \delta, N+M)-a(x, s, \delta, M) \geq \operatorname{ctr}(L(x) N) \geq c \mu \operatorname{tr} N,
$$

for all $N \in S^{n}, N \geq 0$. Let us consider the following problem:

$$
\begin{aligned}
& u \in W^{2, p}(\Omega) \cap W_{0}^{1, p}(\Omega), \\
& a\left(x, u, D u, D^{2} u\right)=f(x), \text { for a.e. } x \in \Omega .
\end{aligned}
$$

Our main result is the following theorem.

Theorem IV.2 If the function a satisfies the assumptions (a1)(a5) then the problem (4.8) has a unique solution $u_{f}$ for every $f \in$ $L^{p}(\Omega)$. Moreover, there exists $C>0$ such that the following estimate holds

$$
\left\|u_{f}\right\|_{W^{2, p}} \leq C\|f\|_{L^{p}}
$$

for all $f \in L^{p}(\Omega)$.

For the proof of Theorem 4.2 we need the following lemmas.

Lemma IV.1 ( [21], Theorem 9.15, Lemma 9.17, p. 242)

The linear elliptic operator $B: W^{2, p}(\Omega) \cap W_{0}^{1, p}(\Omega) \rightarrow L^{p}(\Omega)$ (defined by (4.5)) is well defined and it is a homeomorphism.

Moreover, there exists $\tilde{C}>0$ such that

$$
\|u\|_{W^{2, p}} \leq \tilde{C}\|B u\|_{L^{p}} .
$$


Let us consider now the mapping

$$
\begin{aligned}
& A: W^{2, p}(\Omega) \cap W_{0}^{1, p}(\Omega) \rightarrow L^{p}(\Omega) \\
& A u(x)=a\left(x, u, D u, D^{2} u\right) .
\end{aligned}
$$

Lemma IV.2 A is well defined and continuous.

Proof. Using the condition (a4) we get that

$$
\left|a\left(x, u, D u, D^{2} u\right)\right| \leq \alpha|u(x)|+\beta|D u(x)|_{n}+\gamma\left|D^{2} u\right|_{n^{2}},
$$

or, if $n<2 p$,

$$
\left|a\left(x, u, D u, D^{2} u\right)\right| \leq \alpha(|u(x)|)\left(\beta|D u(x)|_{n}^{s}+\gamma\left|D^{2} u\right|_{n^{2}}\right),
$$

for every $u \in W^{2, p}(\Omega) \cap W_{0}^{1, p}(\Omega)$.

When $n<2 p$, the Sobolev imbedding theorem ([1]) assures that $W^{2, p}(\Omega) \subset C(\bar{\Omega})$ and $W^{2, p}(\Omega) \subset W^{1, q}(\Omega)$ for every $q \geq 1$.

Then, in both cases the right side of this inequality is an $L^{p}$-function, and we can deduce that $A$ is well-defined and continuous.

Lemma IV.3 $A$ is weakly-near to $B$.

Proof. $A$ is continuous with respect to $B$ because $A$ and $B^{-1}$ are continuous.

We will prove that $A$ is strongly-accretive with respect to $B$.

The normalized duality map of the Banach space $L^{p}(\Omega)$ is

$$
J: L^{p}(\Omega) \rightarrow L^{q}(\Omega), \quad J u(x)=u(x)|u(x)|^{p-2}\|u\|^{2-p}
$$

where $\frac{1}{p}+\frac{1}{q}=1$.

Using (4.7) we obtain that, for every $u, v \in W^{2, p}(\Omega) \cap W_{0}^{1, p}(\Omega)$ :

$$
\begin{aligned}
& \langle A u-A v, J(B u-B v)\rangle= \\
= & \|B(u-v)\|^{2-p} . \\
\cdot & \int_{\Omega}\left[a\left(x, u, D u, D^{2} u\right)-a\left(x, v, D v, D^{2} v\right)\right] . \\
\cdot & B(u-v) \cdot|B(u-v)|^{p-2} d x \geq \\
\geq & c|| B(u-v)\left\|^{2-p} \int_{\Omega}|B(u-v)(x)|^{p} d x=c|| B(u-v)\right\|^{2} .
\end{aligned}
$$


Thus

$$
\langle A u-A v, J(B u-B v)\rangle \geq c\|B u-B v\|^{2} .
$$

Now we can prove Theorem 4.2 :

Proof. Because $A$ is weakly-near to $B$ and $B$ is bijective, by Proposition 2.1, we have that $A$ is bijective. Then the problem (4.8) has a unique solution for every $f \in L^{p}(\Omega)$.

In (4.12) we put $v=0$ and the unique solution $u$ of $A u=f$. Then we obtain

$$
\|B u\|^{2} \leq \frac{1}{c}\langle f, J(B u)\rangle
$$

So,

$$
\|B u\|_{L^{p}} \leq \frac{1}{c}\|f\|_{L^{p}}
$$

Using (4.10) we can deduce the estimate (4.9).

In [34] the following problem is also considered

$$
\begin{aligned}
& u \in H^{2}(\Omega) \cap H_{0}^{1}(\Omega), \\
& a\left(x, D^{2} u\right)+g(x, u)=f(x), \text { a.e. } x \in \Omega .
\end{aligned}
$$

The function $g: \Omega \times \mathbb{R} \rightarrow \mathbb{R}$ is measurable in $x \in \Omega$ and there exists $0<c<\lambda_{1}$ (where $\lambda_{1}$ is the smallest eigenvalue of the Laplace operator $-\Delta$ ) such that, for all $r, s$ in $\mathbb{R}$ and for almost every $x$ in $\Omega$

(i) $g(x, 0)=0$,

(ii) $0 \leq[g(x, r)-g(x, s)](r-s)$,

(iii) $|g(x, r)-g(x, s)| \leq c|r-s|$.

Existence and uniqueness results for problem (4.13) were obtained for sufficiently small constants $c$.

We give a similar result for the problem

$$
\begin{aligned}
& u \in H^{2}(\Omega) \cap H_{0}^{1}(\Omega), \\
& a\left(x, u, D u, D^{2} u\right)-g(x) u=f(x), \text { a.e. } x \in \Omega .
\end{aligned}
$$

We assume that $g$ satisfies either (g1) or (g2) stated below: 
(g1) $g \in H^{2}(\Omega) \cap L^{\infty}(\Omega), B g(x) \leq 0$ and $g(x) \geq 0$ a.e. $x \in \Omega$.

(g2) $g(x) \equiv g \in(-\infty, 0)$ and $-g<c \cdot \lambda_{1}(-B)$, where $\lambda_{1}(-B)$ is the first eigenvalue of $(-B)$.

In addition to previous assumptions for the linear elliptic operator $B$, we consider that it has the divergence form

$$
B u=\sum_{i, j=1}^{n} \frac{\partial}{\partial x_{j}}\left(l_{i j}(x) \frac{\partial u}{\partial x_{i}}\right)-l_{0}(x) u, l_{i j}=l_{j i} \in C^{1}(\Omega)
$$

Lemma IV.4 If $(g 1)$ is satisfied then for every $u \in H^{2}(\Omega) \cap H_{0}^{1}(\Omega)$ the following relation holds

$$
-\int_{\Omega} g(x) u(x) B u(x) d x \geq 0 .
$$

If (g2) is satisfied then for every $u \in H^{2}(\Omega) \cap H_{0}^{1}(\Omega)$ the following relation holds

$$
-\int_{\Omega} g \cdot u(x) B u(x) d x \geq \frac{g}{\lambda_{1}(-B)} \int_{\Omega}|B u(x)|^{2} d x .
$$

Proof. If (g1) holds then we have:

$$
\begin{aligned}
& -\int_{\Omega} g(x) u(x) B u(x) d x= \\
= & -\sum_{i, j=1}^{n} \int_{\Omega} g u \frac{\partial}{\partial x_{j}}\left(l_{i j} \frac{\partial u}{\partial x_{i}}\right) d x+\int_{\Omega} g l_{0} u^{2} d x= \\
= & \sum_{i, j=1}^{n} \int_{\Omega} l_{i j} \frac{\partial(g u)}{\partial x_{j}} \frac{\partial u}{\partial x_{i}} d x+\int_{\Omega} g l_{0} u^{2} d x= \\
= & \sum_{i, j=1}^{n} \int_{\Omega} l_{i j} \frac{\partial g}{\partial x_{j}} \frac{\partial u}{\partial x_{i}} u d x+\sum_{i, j=1}^{n} \int_{\Omega} l_{i j} g \frac{\partial u}{\partial x_{j}} \frac{\partial u}{\partial x_{i}} d x+\int_{\Omega} g l_{0} u^{2} d x \geq \\
\geq & \frac{1}{2} \sum_{i, j=1}^{n} \int_{\Omega} l_{i j} \frac{\partial g}{\partial x_{j}} \frac{\partial u^{2}}{\partial x_{i}} d x+\int_{\Omega} g l_{0} u^{2} d x= \\
= & -\frac{1}{2} \int_{\Omega} u^{2} B g d x+\frac{1}{2} \int_{\Omega} g l_{0} u^{2} d x \geq 0 .
\end{aligned}
$$


If (g2) is satisfied then:

$$
\begin{gathered}
-\int_{\Omega} g \cdot u(x) B u(x) d x=-g\langle u, B u\rangle_{L^{2}}= \\
=-(-g)|\langle u,-B u\rangle| \geq-(-g) \frac{1}{\lambda_{1}(-B)}\|B u\|_{L^{2}}^{2} .
\end{gathered}
$$

We used that $\langle u, B u\rangle \leq 0$, and the inequality $|\langle v, T v\rangle| \leq\left|\lambda_{1}(T)\right| \cdot|v|^{2}$ for the linear, self-adjoint and completely continuous operator $T=$ $(-B)^{-1}: L^{2}(\Omega) \rightarrow L^{2}(\Omega)$ (see $\left.[29]\right)$.

Theorem IV.3 If the conditions (a1)-(a5) and (g1) or (g2) hold then there exist $C>0$ and a unique solution $u_{f}$ of (4.14) for every $f \in L^{2}(\Omega)$ such that

$$
\|u\|_{H^{2}} \leq C\|f\|_{L^{2}} .
$$

Proof. Let us consider the mapping $\tilde{A}: H^{2} \cap H_{0}^{1}(\Omega) \rightarrow L^{2}(\Omega)$, defined by $\tilde{A} u(x)=A u(x)-g(x) u$, where $A$ is given by (4.11). Arguing as above, we get that $\tilde{A}$ is continuous with respect to $B$. Also, $\tilde{A}$ is strongly-accretive with respect to $B$, as follows by the following relations (we use Lemma 4.4 and (4.12)):

$$
\begin{aligned}
& \langle\tilde{A} u-\tilde{A} v, B u-B v\rangle= \\
= & \langle A u-A v, B u-B v\rangle-\int_{\Omega} g(x)(u-v)(x) B(u-v)(x) d x \geq \\
\geq & \left(c+\varepsilon \cdot \frac{g}{\lambda_{1}(-B)}\right)\|B u-B v\|_{L^{2}}^{2},
\end{aligned}
$$

where $\varepsilon=0$ if $(g 1)$ holds and $\varepsilon=1$ if ( $g 2)$ holds.

Then $\tilde{A}$ is weakly-near $B$, which is bijective. Thus $\tilde{A}$ is bijective and the existence and unicity of the solution of (4.14) follows. The estimation can be obtained as in the proof of Theorem 4.2.

\section{References}

1. Adams R. A., Sobolev Spaces, Academic Press, New York/San Francisco/London, 1975. 
2. W. Alt \& I. Kolumbán, Implicit function theorems for monotone mappings, Kybernetika 1993, 29, 210-221.

3. J.P. Aubin \& H. Frankowska, Set-Valued Analysis, Birkhauser, Boston (1990).

4. H. Brézis, Analyse Fonctionnelle. Theorie et Applications, Masson, Paris, 1983.

5. F.E. Browder, Nonlinear equations of evolution and nonlinear accretive operators in Banach spaces, Bull. AMS 1967, 73, 867874 .

6. F.E. Browder, Generalization of accretivity for nonlinear mappings of Banach spaces, Bull. AMS 1972, 78, 93-98.

7. A. Buică, Data dependence theorems on coincidence problems, Studia Univ. Babes-Bolyai, 1996, 41, 33-40.

8. A. Buică, Existence of strong solutions of fully nonlinear elliptic equations, submitted.

9. L.A. Caffarelli, X. Cabre, Fully Nonlinear Elliptic Equations, AMS Colloquium Publications, 1995, vol. 43.

10. S. Campanato, Further contribution to the theory of near mappings, Le Matematiche 1993, 48, 183-187.

11. S. Campanato, A Cordes type condition for nonlinear variational systems, Rend. Acc. Naz. delle Sc. 1989, 107, 307-321.

12. C.E. Chidume, Approximation of fixed points of strongly pseudo-contractive mappings, Proc. of AMS 1994, 120, 545551.

13. C.E. Chidume \& M.O. Osilike, Iterative solutions of nonlinear accretive operator equations in arbitrary Banach spaces, Nonlinear Analysis 1999, 36, 863-872.

14. C.E. Chidume \& M.O. Osilike, Approximation of a solution for a $K$-positive definite operator equation, J. Math. Anal. Appl. 1997, 210,1-7.

15. C.E. Chidume \& H. Zegeye, Approximation of zeros of maccretive operators, Nonlinear Analysis, 1999, 37, 81-96.

16. B. Chuanzi, Approximation of a solution for a $K$-positive definite operator equation in uniformly smooth separable Banach space, J. Math. Anal. Appl. 1999, 236, 236-242.

17. K. Deimling, Zeros of accretive operators, Manuscripta Mathe- 
matica 1974, 13, 365-375.

18. K. Deimling, Nonlinear Functional Analysis, Springer-Verlag, 1985.

19. A. Domokos, Implicit function theorems for m-accretive and locally accretive set-valued mappings, Nonlinear Analysis 2000, 41, 221-241.

20. A.L. Dontchev \& W.W. Hager, Implicit functions, Lipschitz maps and stability in optimization, Math. Oper. Res. 1994, 19, 753-768.

21. D. Gilbarg \& N.S. Trudinger, Elliptic Partial Differential Equations of Second Order, Springer-Verlag, 1983.

22. W.A. Kirk, A fixed point theorem for local pseudo-contractions in uniformly convex spaces, Manuscripta Mathematica 1979, 30, 89-102.

23. W.A. Kirk \& M.S. Anderson, A coincidence theorem for mappings satisfying local conditions, Boll. UMI 1983, 2, 159-170.

24. L. Lishan, Ishikawa-type and Mann-type iterative process with errors for constructing solutions of nonlinear equations involving m-accretive operators in Banach spaces, Nonlinear Analysis 1998, 34, 307-317.

25. C. Moore, The solution by iteration of nonlinear equations involving $\Psi$-strongly accretive operators, Nonlinear Analysis 1999, 37, 125-138.

26. C. Morales, Pseudo-contractive mappings and the LeraySchauder boundary condition, Comment. Math. Univ. Carolinae 1979, 20, 745-757.

27. C. Morales, Zeros for strongly accretive set-valued mappings, Comment. Math. Univ. Carolinae 1986, 27, 455-469.

28. C. Morales, Surjectivity theorems for multi-valued mappings of accretive type, Comment. Math. Univ. Carolinae 1985, 26, 397-413.

29. R. Precup, Ecuatii cu Derivate Partiale, (in Romanian), Transilvania Press, Cluj, 1997.

30. S. Reich \& R. Torrejon, Zeros of accretive operators, Comment. Math. Univ. Carolinae 1980, 21, 619-625.

31. S.M. Robinson, Strongly regular generalized equations, Math. 
Oper. Res. 1980, 5, 43-62.

32. S.M. Robinson, An implicit function theorem for a class of nonsmooth functions, Math. Oper. Res. 1991, 16, 292-309.

33. S. Salsa, Lezioni su equazioni ellitiche del secondo ordine, Preprint n.21/R, 1998, Politecnico di Milano.

34. A. Tarsia, Differential equations and implicit function: a generalization of the near operators theorem, Topol. Meth. Nonlin. Anal. 1998, 11, 115-133.

35. A. Tarsia, Some topological properties preserved by nearness between operators and applications to PDE, Czech. Math. J. 1996, 46, 607-624.

36. N.D. Yen, Holder continuity of solutions to a parametric variational inequality, Appl. Math. Optim. 1995, 31, 245-255.

37. E. Zeidler, Nonlinear Functional Analysis and its Applications, vol.II/b, Springer-Verlag, 1990.

38. H.-Y. Zhou, Stable iteration procedures for strong pseudocontractions and nonlinear equations involving accretive operators without Lipschitz assumption, J. Math. Anal. Appl. 1999, 230, $1-10$. 